\title{
Transabdominal Ultrasound-guided Follicular Aspiration in Case of Müllerian Anomaly with Ovarian Malposition
}

\author{
${ }^{1}$ Vineet V Mishra, ${ }^{2}$ Sumesh Choudhary, ${ }^{3}$ Preeti A Goyal, ${ }^{4}$ Rohina Aggarwal
}

\begin{abstract}
Objectives: To describe the approach of transabdominal ultrasound-guided oocyte retrieval in a patient of Müllerian anomaly and ovarian malposition.

Design: Case report.

Setting: A tertiary referral reproductive medicine unit.

Patient: A 23-year-old patient with Müllerian anomaly (right unicornuate with left rudimentary horn uterus) and ovarian malposition.

Intervention: Controlled ovarian hyperstimulation, transabdominal ultrasound-guided oocyte retrieval.
\end{abstract}

Main outcome measure: Total and mature oocytes retrieved, fertilization rate, embryo number, and quality.

Results: Five oocytes were retrieved transabdominally, using percutaneous needle puncture under ultrasound guidance.

Conclusion: In in vitro fertilization protocols, patients with Müllerian anomaly with vaginally inaccessible ovaries, transabdominal ultrasound-guided follicular aspiration is a safe and efficacious alternative technique for oocyte retrieval.

Keywords: Ovarian malposition, Oocyte retrieval, Transabdominal follicular aspiration.

How to cite this article: Mishra VV, Choudhary S, Goyal PA, Aggarwal R. Transabdominal Ultrasound-guided Follicular Aspiration in Case of Müllerian Anomaly with Ovarian Malposition. J South Asian Feder Obst Gynae 2017;9(1):53-55.

Source of support: Nil

Conflict of interest: None

Date of received: 10 August 2016

Date of acceptance: 17 November 2016

Date of publication: January 2017

\section{INTRODUCTION}

Müllerian duct anomalies include a spectrum of disorders resulting from abnormal development or fusion of

\footnotetext{
${ }^{1}$ Professor and Head, ${ }^{2,4}$ Assistant Professor, ${ }^{3}$ Senior Resident

${ }^{1-4}$ Department of Obstetrics and Gynecology, Institute of Kidney Disease and Research Centre, Ahmedabad, Gujarat, India

Corresponding Author: Vineet V Mishra, Professor and Head Department of Obstetrics and Gynecology, Institute of Kidney Disease and Research Centre, Ahmedabad, Gujarat, India Phone: +917922687038, e-mail: vineet.mishra.ikdrc@gmail.com
}

the paramesonephric ducts. Although ovaries develop from the genital ridge independently of the mesonephric (Wolffian) and paramesonephric (Müllerian) ducts, descent is governed by the gubernaculum, which attaches the inferior ovarian pole to the uterus, eventually forming the utero-ovarian and round ligaments. Arrested development of any of these structures may result in failure of the ovaries to enter the true pelvis. ${ }^{1}$

The incidence of Müllerian duct anomalies is estimated to be $1 \%$ of the general population and up to $25 \%$ of infertile women. ${ }^{2}$ Although renal anomalies are commonly associated with Müllerian anomalies, a higher incidence of incomplete ovarian descent or ovarian malposition has been seen in these women. ${ }^{3}$ Transvaginal ultrasoundguided follicular aspiration has become the standard technique in women undergoing oocyte retrieval during in vitro fertilization (IVF). But in women with ovarian malposition, ovaries are not accessible transvaginally. In past, either laparoscopy was performed for oocyte retrieval in such cases or these women were not considered for IVF. Transabdominal ultrasound-guided oocyte retrieval is an alternative method which avoids the increased time and risk of laparoscopy. ${ }^{4,5}$ We describe transabdominal ultrasound-guided follicular aspiration in a woman who was diagnosed as a case of Müllerian anomaly with one ovary not accessible by transvaginal ultrasound.

\section{CASE REPORT}

A 23-year-old woman visited our IVF center with history of secondary infertility for last 2 years. She was married for three and half years with history of one first trimester spontaneous abortion. Her menstrual cycle was normal. Her general, systemic, and gynecological examination was found to be normal. On transvaginal ultrasonography with 4 to $8 \mathrm{MHz}$ transvaginal probe (voluson E8), uterus was normal with nonvisualization of bilateral ovaries. The right ovary was better visualized on transabdominal ultrasound with 2 to $5 \mathrm{MHz}$ transabdominal probe (voluson E8) with polycystic morphology and located lateral to the right side of pelvic brim. But the left ovary could not be visualized even with transabdominal ultrasound. She had a single kidney on the right side.

She was diagnosed as a case of Müllerian anomaly on diagnostic hysterolaparoscopy with right unicornuate uterus with left rudimentary horn and distorted tubo-ovarian 
relationship on both sides. The right ovary was located high up on the lateral pelvic wall, and the left ovary was located very high up on lateral pelvic wall above the insertion of left infundibulopelvic ligament. The right fallopian tube was elongated, and the left fallopian tube was stretched over lateral pelvic wall. The left round and ovarian ligaments were absent. Endometriotic spots were seen in pouch of douglas. On chromopertubation, there was free spill present on the right side and absent on the left side. Postoperatively, she was given three injections of leuprolide acetate $3.75 \mathrm{mg} \mathrm{IM}$ at an interval of 4 weeks. Her day 2 hormonal profile revealed normal ovarian function with S. FSH - 6.02 IU/L, S. LH-9.33 IU/L, S. TSH $0.79 \mathrm{mIU} / \mathrm{mL}$, S. Prolactin - $17.53 \mathrm{IU} / \mathrm{L}$, and S. Estradiol $53 \mathrm{pg} / \mathrm{mL}$. Husband's semen analysis was found to be within normal limits. She underwent three cycles of intrauterine inseminations with ovarian stimulation done using tab clomiphene citrate $50 \mathrm{mg}$ and recombinant follicle stimulating hormone (FSH). But no positive result was found, and the couple was counseled for IVF.

The patient underwent controlled ovarian stimulation using long downregulation protocol. She was given injection leuprolide acetate $0.5 \mathrm{cc} \mathrm{s} / \mathrm{c}$ daily from day 21 . From day 2, injection recombinant FSH was started at a dose of $225 \mathrm{IU} /$ day IM. Stimulated right ovary was well visualized on transvaginal ultrasound and left ovary was visualized on transabdominal ultrasound. Human chorionic gonadotropin (10000 IU IM) was given as trigger on day 11 . Oocyte retrieval was done 36 hours later under short general anesthesia; 12 follicles were visualized in the right ovary by transvaginal ultrasound, and 7 follicles were visualized in left ovary by transabdominal ultrasound. Right ovarian follicular aspiration was done with a 24-cm long, 17-guage aspiration needle (reproline) via transvaginal ultrasound (4-8 $\mathrm{MHz}$ transvaginal probe). Left ovarian follicular aspiration was done by transabdominal ultrasound-guided percutaneous route (2-5 MHz transabdominal probe) through left lower quadrant of the abdominal wall using the same aspiration needle. Aspiration needle was briskly thrust through the abdominal wall directly in to the ovary. All follicles were aspirated through two punctures (Fig. 1).

From the right ovary, nine oocytes were retrieved and from left ovary five oocytes were retrieved. Out of five oocytes obtained from left ovary four oocytes were mature and one was immature. The total number of mature oocytes was 10 and immature oocytes were four. A total 10 grade A embryos were obtained. The patient got discharged without any complications. She underwent frozen cycle embryo transfer. At 37 weeks, she underwent cesarean section and gave birth to a healthy female child of $2.7 \mathrm{~kg}$.

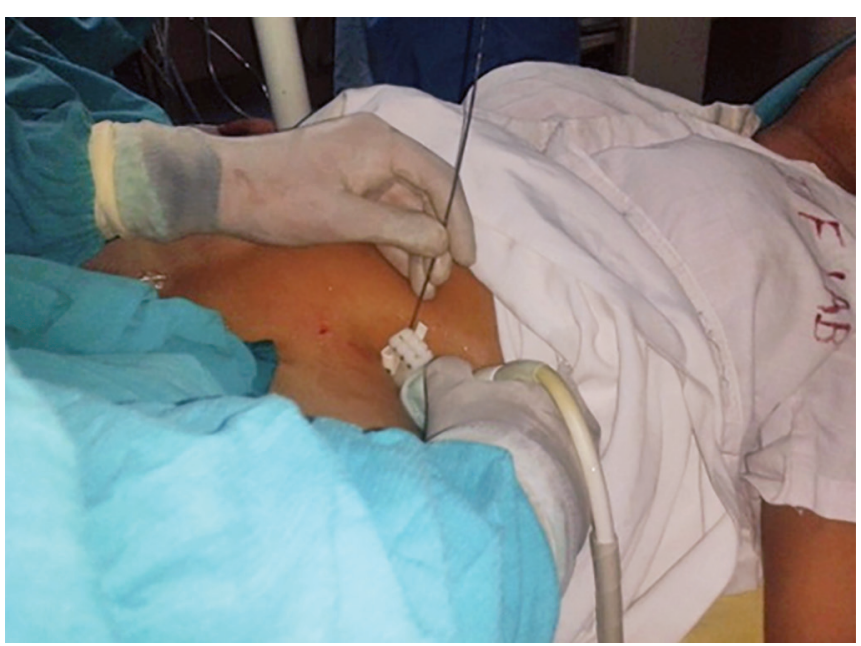

Fig. 1: Technique of transabdominal ultrasound-guided follicular aspiration

\section{DISCUSSION}

Transvaginal ultrasound-guided follicular aspiration is the most preferred technique for oocyte retrieval in IVF protocols. But when we come across such patients with abnormal pelvic anatomy, in whom ovaries are not accessible with transvaginal sonography, then transabdominal ultrasound-guided follicular aspiration is the most feasible approach alternative to laparoscopy. A study by Barton et $\mathrm{al}^{4}$ demonstrated that the number of mature oocyte retrieved was comparable by both approaches, and there was no increase in damage to oocytes noticed during transabdominal route.

Despite the feasibility of this approach, there are some limitations of transabdominal route. This has been seen that gynecologist is more skilled in doing transvaginal follicular monitoring and aspiration due to the frequency of its use. ${ }^{4,6}$ Multiple punctures are often required for transabdominal aspiration due to diminished elasticity of abdominal wall leading to increased patient discomfort and skin scarring. Rate of infections are very less. Patients need counseling for all these limitations.

\section{CONCLUSION}

In IVF protocols, patients with Müllerian anomaly with inaccessible ovaries vaginally, transabdominal ultrasoundguided follicular aspiration is a safe and efficacious alternative technique for oocyte retrieval.

\section{REFERENCES}

1. Allen JW, Cardall S, Kittijarukhajorn M, Siegel CL. Incidence of ovarian maldescent in women with Müllerian duct anomalies: evaluation by MRI. AJR Am J Roentgenol 2012 Apr;198(4):W381-W385. 
2. Troiano RN, McCarthy SM. Müllerian duct anomalies: imaging and clinical issues. Radiology 2004 Oct;233(1):19-34

3. Dabirashrafi $\mathrm{H}$, Mohammad K, MoghadamiTabrizi N. Ovarian malposition in women with uterine anomalies. Obstet Gynecol 1994 Feb;83(2):293-294

4. Barton SE, Politch JA, Benson CB, Ginsburg ES, Gargiulo AR. Transabdominal follicular aspiration for oocyte retrieval in patients with ovaries inaccessible by transvaginal ultrasound. Fertil Steril 2011 Apr;95(5):1773-1776.
5. Raziel A, Vanknin Z, Schachter M, Strassburger D, Herman A, Ron-El R, Friedler S. Ultrasonographic-guided percutaneous transabdominal puncture for oocyte retrieval in a rare patient with Rokitansky syndrome in an in vitro fertilization surrogacy program. Fertil Steril 2006 Dec;86(6): 1760-1763.

6. Damario MA. Transabdominal-transperitoneal ultrasoundguided oocyte retrieval in a patient with mullerain agenesis. Fertil Steril 2002 Jul;78(1):189-191. 